REVISTA DE LOGOPEDIA, FONIATRÍA Y AUDIOLOGÍA

\title{
Medida de la Inteligibilidad en el Habla Disártrica
}

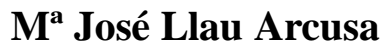 \\ Logopeda y Psicóloga. Centro de Psicología y Logopedia. Castellón de la Plana
}

\section{Dr. Julio González Álvarez}

Profesor Titular de Psicología del Lenguaje.

Departamento de Psicología Básica, Clínica y Psicobiología,

Universidad Jaume I de Castellón de la Plana

PALABRAS DE CABECERA: INTELIGIBILIDAD DEL HABLA DISÁRTRICA

Trabajo financiado por Fundació Caixa Castelló-Bancaixa-Universitat Jaume I, Castellón de la Plana (Proyecto P1A99-01), y por el Ministerio de Ciencia y Tecnología, (Proyecto $\mathrm{I}+\mathrm{D}$, convocatoria 2003, BSO2003-01002/PSCE).

Correspondencia a:

D María José Llau Arcusa

Ramón y Cajal, 12, $3^{a}$

12002 Castellón

email: mjosllau@correo.cop.es

teléfono: 964-269600 


\section{RESUMEN}

El término disartria designa un conjunto de alteraciones del habla causadas por daño neurológico. Tratándose del trastorno adquirido del habla más frecuente, la medida de la inteligibilidad en los pacientes disártricos es de gran importancia tanto para el diagnóstico clínico como en la investigación de base. Sin embargo, una medida objetiva y fiable de la inteligibilidad es difícil al tratarse de una magnitud relativa dependiente de variables concretas del proceso de comunicación. El presente trabajo revisa el concepto de inteligibilidad y los principales procedimientos de medida aplicados para el habla disártrica (escalas de evaluación y tests de identificación). Los autores defienden la necesidad de tests estandarizados que proporcionen no sólo un índice general de severidad, sino que analicen también los contrastes fonético-acústicos vulnerados en cada caso, en línea con el test de Kent et al. (1989) para el inglés. En términos ideales, un test de este tipo debería ser capaz de identificar las principales razones que causan el déficit de inteligibilidad y suministrar información útil para el tratamiento logopédico.

Palabras clave: inteligibilidad, disartria, habla, tests, medida. 


\section{SUMMARY}

Dysarthria is the most commonly acquired speech disorder and it consists of speech abnormalities caused by neuromuscular disorders. The measurement of intelligibility in dysarthric individuals is a major concern in clinical assessment and in research on dysarthria. However, an objective and reliable measurement is complicated by the fact that intelligibility is a relative quantity that depends on specific variables from the process of communication. The present paper reviews the concept of speaker intelligibility and the main measuring procedures as they have been applied to dysarthric speech (scaling procedures and item identification tests). The authors defend the necessity of standardized tests designed to provide not only an overall index of severity, but also the specific acoustic-phonetic contrasts that contribute significantly to speech intelligibility, in line with Kent et al. (1989) for English. Ideally, the results of a test of this type should identify reasons for an intelligibility deficit and they should provide useful information for speech management.

Key words: intelligibility, dysarthria, speech, tests, measure. 


\section{Introducción}

Una secuela frecuente de las lesiones neurológicas es la aparición de dificultades de tipo disártrico en el lenguaje del paciente. Con el término disartria se designa colectivamente a un grupo de trastornos causados por alteraciones del control muscular sobre los mecanismos del habla a consecuencia de un daño en el sistema nervioso central o periférico. Se trata de dificultades en la comunicación oral debidas a parálisis, debilidad o falta de coordinación de los músculos que participan en la producción del lenguaje (Darley, Aronson y Brown, 1975;

Duffy, 1995). Son ya clásicos los siete tipos establecidos por la norteamericana Clínica Mayo en los años 70, y reactualizados después por Duffy (1995). Conviene distinguir entre disartrias de desarrollo, por lesión fetal/perinatal o hipodesarrollo ligado a cuadros hereditarios que se expresan durante la infancia, y disartrias adquiridas, cuando éstas sobrevienen una vez finalizado el desarrollo lingüístico. En la presente revisión nos centraremos fundamentalmente sobre estas últimas.

En la edad adulta, las causas más frecuentes de la disartria son ictus o accidentes cerebrovasculares (ACV); traumatismos craneo-encefálicos (TCE), sobre todo a consecuencia de accidentes de tráfico; tumores cerebrales; y enfermedades neurodegenerativas, como Parkinson, esclerosis múltiple, esclerosis lateral amiotrófica, enfermedad de Friedreich, corea, etc. Conviene diferenciar este último grupo asociado a una enfermedad progresiva de los restantes procesos en los que cabe esperar una plena recuperación o una mejoría hasta un nivel estable de capacidad. Dentro de las enfermedades neurodegenerativas, también es interesante distinguir entre las que cursan con un deterioro rápido e inexorable, y aquellas en las que el declive puede durar muchos años, con posibles periodos de remisión (Miller, 2001).

Un estudio frecuentemente citado de la Clínica Mayo ofrecía los siguientes porcentajes en la distribución de trastornos de comunicación adquiridos por causa neurológica durante los 
años 1987-90 (Duffy, 1995): disartrias (46.3\%); afasias (27.1\%); apraxias del habla (4.6\%); otros trastornos cognitivo-lingüísticos (13.0\%); otros trastornos neurogénicos $(9.0 \%)$. Tratándose del trastorno adquirido del habla más frecuente (Enderby, 1983), llama la atención el hecho de no disponer aún en nuestro ámbito de un instrumento de evaluación estandarizado que ofrezca una medida cuantitativa del grado de severidad y tipo de afectación del habla disártrica y su repercusión en el proceso de comunicación.

Dentro del impacto general que los trastornos disártricos causan en el lenguaje del paciente (pérdida de naturalidad y expresividad, reducción de la fluidez, distorsión de la cualidad vocal, etc.) destaca la merma que en muchos casos se produce en su competencia comunicativa a causa de la reducción de la inteligibilidad del habla. Tanto en la clínica como en la investigación interesa disponer de técnicas de evaluación que proporcionen medidas consistentes y válidas de la inteligibilidad del habla disártrica. En opinión de Yorkston y Beukelman (1981) y otros autores, su utilidad se basa fundamentalmente en:

a.- Se trataría de la medida que mejor contribuye a la obtención de un índice global de severidad y la posibilidad de comparación entre pacientes disártricos.

b.- Su valor sería central en la comparación de muestras de habla de un mismo paciente, como estimador objetivo del progreso de un tratamiento (o avance del deterioro en enfermedades progresivas).

c.- Tiene una gran validez aparente y es fácilmente comunicable a la familia y otros profesionales.

d.- La inteligibilidad se trata, en definitiva, de la variable más estrechamente relacionada con la transmisión de información en el proceso de comunicación de la persona disártrica (Beukelman y Yorkston, 1979). 


\section{Concepto de inteligibilidad y su medida.}

La inteligibilidad del habla ocupa un lugar central en la constelación de dificultades que genera la disartria, dada su importancia e incidencia directa en el proceso de comunicación del sujeto. Puede definirse como el grado en que un mensaje intencionado del hablante es recuperado por el oyente (Kent, Weismer, Kent, y Rosenbeck, 1989). O más concretamente, el grado en que se acoplan la intención del hablante y la respuesta del oyente (Schiavetti, 1992). En el esquema general de la comunicación, donde la información fluye desde el hablante al oyente a través de un canal, la inteligibilidad puede disminuir por causas localizadas en cualquiera de los tres elementos básicos. Cuando la dificultad se sitúa en la fuente o hablante, la inteligibilidad se ve afectada por deterioro de la señal de habla, como así ocurre en el lenguaje disártrico, habla del sordo, graves disfonías, etc. Si la dificultad se ubica en el receptor u oyente, estamos hablando fundamentalmente de las sorderas o hipoacusias. El canal o sistema de transmisión también juega un papel importante en la inteligibilidad del lenguaje (por ejemplo, teléfono, ambiente ruidoso, superposición de conversaciones, etc.). Gran parte del conocimiento generado sobre la inteligibilidad del habla humana proviene de estudios centrados sobre el canal; concretamente, nos estamos refiriendo al extenso programa de investigaciones dirigidas por los ingenieros de telecomunicaciones en los primeros años de la telefonía en EE.UU. (ver Fletcher y Allen, 1995, una revisión de 33 años de trabajo en los laboratorios Bell bajo la dirección del ingeniero Harvey Fletcher).

Cuando se pretende medir la inteligibilidad del habla, no hay que olvidar que se trata en realidad de un concepto relativo. En términos operativos, el enfoque clásico define su medida como "el cómputo de las unidades discretas del habla reconocidas correctamente por un oyente" (Flanagan, 1972). Pero este cómputo o proporción de unidades reconocidas va a depender de los distintos parámetros que configuran el proceso de comunicación. Decir que un paciente tiene una inteligibilidad del $45 \%$ no es una afirmación interpretable en sí misma 
si previamente no se especifican las condiciones bajo las cuales se obtuvo ese valor (Kent et al., 1989). El resultado obtenido podría ser otro bien distinto dependiendo de la clase de contenido verbal sometido a prueba (pseudopalabras, palabras, frases, lenguaje espontáneo, etc.), las condiciones acústicas del entorno, el grado de familiaridad que el oyente tiene con el sujeto o con otros sujetos disártricos, etc. En otras palabras, la inteligibilidad del habla humana es dependiente del contexto y puede ser considerada un concepto pragmático (Connolly, 1986).

Los intentos por medir la inteligibilidad del habla se han basado principalmente en dos métodos muy distintos que pasamos a considerar. Por una parte, se han aplicado procedimientos derivados de las escalas de evaluación subjetiva y, por otra, los que consisten en la identificación de estímulos verbales.

\section{Escalas de Evaluación.}

Los procedimientos de "escalado", o basados en escalas de evaluación, constituyen una metodología habitual en el estudio del comportamiento humano. En logopedia se usan para valorar diversas dimensiones de la conducta verbal, tales como la calidad de la voz, fluidez verbal, grado de disfonía, nivel de tartamudez, etc. Su utilidad descansa preferentemente sobre dos ventajas (Schiavetti, 1992): en primer lugar, algunos autores consideran que muchas dimensiones relacionadas con los trastornos de comunicación son evaluables más directamente a través de escalas; en segundo lugar, cuentan a su favor la simplicidad y rapidez de uso. Entre las escalas más usadas en disartria, destacan las de la Clínica Mayo, en versión preliminar de Darley, Aronson y Brown (1975) y posteriormente de Joseph Duffy (Duffy, 1995). La escala de Duffy se compone de 10 puntuaciones que van desde 1.-“el habla no es un medio viable de comunicación en ningún tipo de entorno" hasta 10.-“habla normal en todos los entornos, sin restricción de contenido y sin necesidad de aclaración”. Cada puntuación 
contempla 3 dimensiones: entorno en el que se da la comunicación, el contenido, y la eficiencia comunicativa.

La técnica de escalado más conocida es la de los intervalos iguales, mediante la cual el oyente-evaluador debe asignar un número a cada muestra de habla que representa su posición dentro de un continuo de la dimensión evaluada, inteligibilidad en este caso. Suelen emplearse escalas de números impares $(5,7,9)$, para poder contar con un número central, además de los dos extremos. Algunos instrumentos emplean descripciones cualitativas ligadas a cada puntuación (p.e. desde 1-completamente ininteligible a 5-completamente inteligible), mientras que otros incluyen únicamente las puntuaciones, sin que parezca que este aspecto sea clave en las propiedades psicométricas de la escala (Stevens, 1975; Guilford, 1954). Las muestras de habla procedentes de sujetos disártricos con distintos grados de inteligibilidad suelen ser frases o fragmentos de un párrafo previamente grabados que se presentan de forma aleatoria al evaluador. Es práctica común que al principio éste se familiarice con el rango completo de inteligibilidad mediante la presentación preliminar de unos pocos ejemplos que abarque todo el rango de variabilidad.

Otro procedimiento, éste menos habitual, consiste en la estimación directa de la inteligibilidad. Aquí el oyente no está restringido a una cantidad prefijada de intervalos y puede juzgar cada estímulo atribuyéndole un número que él considere proporcional a la relación de inteligibilidad entre las muestras. Este procedimiento puede llevarse a cabo con o sin el uso de un estándar o módulo, que sería un estímulo, generalmente de inteligibilidad media, que sirve de referencia básica para contrastar cada muestra de habla.

La investigación ha demostrado repetidamente que la evaluación mediante escalas perceptivas se comporta de modo distinto dependiendo del tipo de magnitud o dimensión que se evalúa (Stevens, 1975). En líneas generales hay dos grandes grupos de dimensiones. Unas son aquellas que constituyen un continuo aditivo (protético), en el que los diversos valores 
que puede adoptar son descritos mejor como grados o cantidades de la magnitud que se van acumulando. Un ejemplo paradigmático es la percepción de la intensidad del sonido. El otro gran grupo son las dimensiones basadas en un continuo sustitutivo (metatético), donde cada valor no se percibe como una acumulación de los anteriores, sino como algo cualitativamente distinto que los sustituye. El ejemplo típico es la percepción del tono de un sonido: un tono agudo no se percibe como "más cantidad" o acumulación de los tonos anteriores, más graves, sino como algo cualitativamente distinto. Los estudios psicofísicos muestran que, mientras una magnitud metatética puede ser evaluada por ambos procedimientos (intervalos y estimación directa), las magnitudes protéticas no deben ser evaluadas por el procedimiento de intervalos, porque los observadores son incapaces de partir un continuum protético en intervalos equivalentes (Snodgrass, 1975; Stevens, 1975). Los datos indican que los evaluadores tienden invariablemente hacia la subdivisión del extremo inferior del continuum en intervalos más pequeños. Ahora bien, parece que la inteligibilidad es una magnitud del tipo protético (Schiavetti, 1992; Schiavetti, Metz y Sitler, 1981), por lo que la escala de intervalos sería una técnica inapropiada para medir la inteligibilidad del habla disártrica, ya que los oyentes serían incapaces de establecer intervalos equivalentes a lo largo de un continuum inteligible-no inteligible. En principio, el uso de una escala ordinal basada en la estimación directa sería más adecuada para medir la inteligibilidad del habla.

\section{Identificación de Estímulos Verbales.}

La identificación o reconocimiento por parte de un oyente de estímulos verbales, generalmente palabras o frases, pronunciadas por un hablante, entronca con los primeros estudios sobre la inteligibilidad del lenguaje. Los comienzos de la telefonía estimularon abundante investigación acerca de las gamas de frecuencias y otras condiciones físicas que mejor transmitían el habla a través de un canal limitado, como es el teléfono. En última 
instancia, más allá de las especificaciones técnicas de los dispositivos, había de ser un oyente humano quien aportara la prueba definitiva acerca de la eficiencia comunicativa de un sistema (y aún lo sigue siendo hoy, pese al esfuerzo por desarrollar algoritmos matemáticos que estimen la inteligibilidad de una señal lingüística). De aquí surgieron procedimientos estandarizados para el inglés -- basados en las normas ANSI-- y parámetros como el conocido índice de articulación. Paralelamente, la tradición norteamericana en el estudio de la sordera ha producido tests para el inglés (listas de palabras y frases CID, SPIN, HINT etc.) con material estandarizado para evaluar la percepción del lenguaje en los sujetos hipoacúsicos. Para el castellano hemos de mencionar el valioso material de Cárdenas y Marrero (1994) que, desgraciadamente, cuenta con dificultades en su distribución actual, o las diferentes listas de palabras seleccionadas para logometría o audiometría verbal - listas de Tato, listas de Villalba para niños (Villalba, Ferrández y Ros, 1999), etc.

Parece que la identificación de estímulos verbales es una técnica de medida de la inteligibilidad más adecuada que la basada en escalas. Esto sería así tanto desde el punto de vista general como en el plano particular de la disartria. Por de pronto, ya no se trata sólo de una estimación subjetiva de la impresión de inteligibilidad que el lenguaje disártrico produce en un observador, sino la demostración objetiva del nivel de percepción del mismo por medio de unos estímulos convenientemente escogidos. Además de la dificultad, antes indicada, de dividir en intervalos iguales una magnitud protética como la inteligibilidad, Nicholas Schiavetti y otros autores señalan algunas razones en este sentido (ver Schiavetti, 1992). En primer lugar, el porcentaje de palabras -u otros estímulos- correctamente reconocidas por un oyente es una métrica más manejable desde la clínica o la investigación y mejor interpretable por otros profesionales (validez aparente). En segundo lugar, la fiabilidad de los resultados es superior a la encontrada en puntuaciones de escalas. Samar y Mezt (1988) encuentran correlaciones de 0.98 tanto intra-observador como entre observadores, claramente 
superiores a las halladas en escalas. Yorkston y Beukelman (1978) también encuentran una diferencia a favor de los métodos de identificación. Finalmente, el empleo de los métodos o tests de identificación facilita la investigación sobre las características acústicas del lenguaje que guardan estrecha relación con su inteligibilidad; cuestión ésta que veremos más adelante. ¿Qué estímulos se usan en los tests de inteligibilidad basados en la identificación? Está claro que la selección correcta de los mismos va a tener una importancia capital en su capacidad de predecir la inteligibilidad en situaciones naturales. Además de fonemas, son frases y sobre todo listas de palabras los elementos verbales más comunes. Yorkston y Beukelman (1978) encuentran una correlación alta entre el reconocimiento de frases y el de palabras por parte de un grupo de sujetos disártricos de diversa severidad. En cuanto a las tareas o procedimientos, hay un repertorio de menor a mayor dificultad, que va desde el reconocimiento entre un conjunto cerrado de elementos posibles, hasta el reconocimiento abierto sin alternativas. Para los casos más graves, en los que la inteligibilidad es muy baja, se ha visto conveniente la introducción de un contexto semántico, por ejemplo, usando estímulos agrupados por familias de palabras - colores, días de la semana, etc.- (Dowden, Yorkston y Stoel-Gammon, 1987; Yorkston y Beukelman, 1981). Para los casos más leves, Yorkston y Beukelman (1981) consideran valiosa la variable velocidad o tasa de habla, medida por el índice de palabras inteligibles por minuto, que discrimina entre pacientes con inteligibilidad próxima al $100 \%$.

\section{Tests de inteligibilidad del habla disártrica.}

En el momento presente no contamos en España con un test estandarizado dirigido a evaluar las diferentes clases de disartrias (ver la reciente revisión de Melle, 2003). Más específicamente, tampoco existe un test estandarizado que reúna un conjunto de estímulos seleccionados para medir la inteligibilidad del lenguaje disártrico y, sobre todo, la detección 
de errores específicos que sean fuente de la reducción de la misma, al estilo del test de Kent (Kent et al., 1989). Hemos de mencionar, no obstante, el reciente test de inteligibilidad de Marc Monfort (Monfort y Juárez, 2001) formado por una lista de 23 palabras y 9 frases, con un propósito general (población infantil y adulta, sordera, disartria, etc.).

En la Tabla 1 se presentan los principales tests o instrumentos del ámbito internacional construidos para medir la inteligibilidad del habla disártrica mediante tareas de identificación. El trabajo pionero de Tikofsky y Tikofsky (1964) agrupó un conjunto de 160 palabras que se redujo después a 50 (Tykofsky, 1970), en función de su capacidad discriminativa entre un grupo de sólo 9 sujetos disártricos. Uno de los descubrimientos que puso de relieve el análisis de ítems, es el valor de las palabras compuestas, al estilo de "salvavidas" o "abrelatas", en la evaluación de la inteligibilidad y en la discriminación entre pacientes más o menos inteligibles. El equipo australiano de Platt se centró en el análisis de los errores de articulación más frecuentes en el habla disártrica, pero esta información luego no se incorporó sistemáticamente en la selección de los estímulos de la prueba (Platt, Andrews, Young, y Quinn, 1980). Los tests ya “clásicos” sobre disartria, el Frenchay (Enderby, 1983) y el Robertson Profile (Robertson, 1982), incluyen, entre otras pruebas que evalúan diversos aspectos de la conducta motora verbal y no verbal, una prueba de inteligibilidad que no ha sufrido un proceso propio de validación y calibración psicométrica. Como instrumentos específicos de inteligibilidad deben citarse los anglosajones Assessment Intelligibility of Dysarthric Speech (AIDS) de Yorkston y Beukelman (1981), con una versión informatizada posterior, el CAID o Computerized Assessment of Intelligibility of Dysarthric Speech (Yorkston y Beukelman, y Traynor 1984) y el Word Intelligibility Test de Kent y colegas (Kent et al., 1989) en su doble versión de elección múltiple y pares de palabras. La novedad que encierra este último es que, como veremos a continuación, va más allá del cálculo de un 
único índice numérico e intenta obtener información sobre las causas de la pérdida de inteligibilidad.

Fuera del ámbito anglosajón se han llevado a cabo algunos trabajos en otros idiomas. Así, hemos de considerar el test de 100 palabras del equipo de Wolfram Ziegler del Instituto MaxPlanck en Alemania (Ziegler, Hartman, y Von Cramon, 1988) y su versión posterior ampliada e informatizada, el Münchner Verständlichkeitsprofil (MVP) o Perfil de Inteligibilidad de Munich (Ziegler y Hartman, 1993), basada en cuatro listas de más de un centenar de palabras. En Francia, Michèle Gentil adapta el test de Kent al francés (Gentil, 1992) y el equipo de Pascal Auzou, en Rouen, adapta al francés el test Frenchay, incluyendo una prueba de inteligibilidad (Auzou et al., 1998). Muy recientemente, desde la Universidad de Hong Kong, el equipo de Tara Whitehill construye un test para evaluar la inteligibilidad de pacientes disártricos de chino cantonés, el Cantonese Single-Word Inteligibility (CSIT) (Whitehill y Ciocca, 2000b), siguiendo el mismo enfoque del test de Kent, pero después de realizar una investigación previa sobre los contrastes afectados por la disartria en dicha lengua (Whitehill y Ciocca, 2000a).

\section{Análisis de la inteligibilidad: el enfoque del test de Kent et al. (1989)}

¿Por qué el habla disártrica es menos inteligible? Hay hablas o voces patológicas con un alto grado de deterioro y pérdida de naturalidad que, sin embargo, conservan casi intacta la capacidad de ser entendidas; mientras que otras, con un deterioro aparentemente menor, son difícilmente comprensibles. ¿Cuáles son los aspectos críticos que separan a unas de otras? En los últimos años ha cobrado fuerza un enfoque explicativo sobre la pérdida de inteligibilidad en el habla disártrica. Es decir, el interés se centra en conocer los principales factores fonético-articulatorios y los rasgos de deterioro de la señal acústica que la hacen menos inteligible al oído de un oyente. Además de su valor diagnóstico, tiene un extraordinario 
interés clínico porque permitiría focalizar los tratamientos sobre aquellas alteraciones que mayor repercusión tienen en el proceso comunicativo. En este sentido, el test elaborado en la Universidad de Wisconsin-Madison por el equipo de Raymond Kent constituye el primer intento sistemático de disponer de un instrumento que no sólo ofrezca un índice global de inteligibilidad, sino que analice en detalle aspectos parciales de la misma (Kent et al., 1989). En la construcción del test se seleccionó un conjunto de estímulos que pusieran a prueba los errores articulatorios y fonéticos más comunes en las disartrias. Para ello, y previo a la construcción de test, se efectuó un exhaustivo análisis de la literatura científica sobre los contrastes fonéticos del inglés que aparecían afectados con mayor frecuencia en distintos cuadros disártricos. Fruto de este análisis se identificó una lista de 19 contrastes o errores de articulación responsables en gran medida de la pérdida de inteligibilidad, tales por ejemplo como: 1.- contraste anterior-posterior en vocales (p.e feed-food), 3.- contraste entre vocales largas y cortas (beat-bit), 5.- contraste sonoro-sordo en consonantes oclusivas iniciales (batpat), 12.- contraste oclusivo-nasal (dot-knot), ó 19.- contraste [r]-[w] (rock-walk). La identificación de estos contrastes se hizo según dos criterios principales:

a.- Vulnerabilidad: los contrastes debían representar rasgos fonéticos vulnerables a las alteraciones que imponen los diferentes tipos de disartria.

b.- Correlatos acústicos: estos contrastes debían poder ser caracterizados a través de una o varias medidas acústicas del habla. Por ejemplo, el contraste 2.- alto-bajo en vocales (feet-fat) puede ser caracterizado por la frecuencia en hertzios del primer formante (F1), que responde a una resonancia del conducto vocal muy sensible al grado de apertura del mismo (y por ende, a la posición alta-baja de la lengua). De esta manera, se dejaba la puerta abierta al estudio objetivo de las causas de pérdida de inteligibilidad de la señal de habla.

El test se presenta en dos versiones: una general que se basa en el procedimiento de elección múltiple y otra dirigida a sujetos más afectados basada en pares de palabras. Los 
estímulos se disponen de forma que permitan aislar los contrastes afectados en el habla de un paciente particular. Por ejemplo, en la versión de elección múltiple, el primer ítem contiene los siguientes 4 estímulos:
a) $\mathrm{bad}$
b) bed
c) bat
d) pat

Una vez grabada la voz de la persona disártrica pronunciando el estímulo correcto (en este caso, $b a d)$, se da a escuchar a uno o varios oyentes y éstos deben elegir entre las cuatro palabras, cuál ha sido la pronunciada por el paciente. En caso de error, se puede determinar el tipo de contraste afectado dependiendo de la elección del oyente. Por ejemplo, si suena como bed, el contraste afectado es 3.- vocal larga-corta; si suena como bat se trata del contraste 5.sonoro-sordo en oclusiva final; si suena pad es el contraste 4.-sonoro-sordo en oclusiva inicial. El conjunto de estímulos del test completo permite poner a prueba todos los contrastes en más de diez ocasiones.

Esta línea de trabajo se ha revelado muy fructífera, tanto por sus resultados prácticos como por su potencialidad para el avance en el conocimiento de las bases de la inteligibilidad y su deterioro en el habla patológica. De hecho, este enfoque ha inspirado la mayor parte de los esfuerzos recientes que se están realizando en otros ámbitos lingüísticos. Ni que decir tiene que un aspecto crucial es la adecuada selección de los contrastes fonéticos por su impacto en la inteligibilidad y el repertorio de éstos está íntimamente ligado a la estructura fonéticofonológica de cada lengua. Por ejemplo, el contraste 3.- vocal larga-corta no tiene sentido en castellano y, al revés, probablemente hay otros contrastes específicos de nuestro idioma $-\mathrm{y}$ por tanto, no presentes en la lista de Kent- de gran repercusión en la inteligibilidad del castellano.

Creemos que no todos los tests de inteligibilidad que siguen este enfoque para otras lenguas distintas del inglés se han visto precedidas de la suficiente investigación empírica sobre los contrastes más afectados en dichas lenguas. Por razones históricas, las disartrias en 
el ámbito anglosajón cuentan con una tradición de estudio y un volumen de literatura que, desgraciadamente, no se ha acumulado en otras lenguas. Algunos trabajos tal vez han aplicado demasiado mecánicamente la aportación de Kent. Por ejemplo, para el francés, Michèle Gentil (Gentil, 1992) se conforma con usar directamente los contrastes ingleses, descartando dos de ellos que no encajan en la fonética francesa. Con este procedimiento no está claro si los que mantiene son los más relevantes para el francés y, por otra parte, podrían faltar otros contrastes específicos potencialmente relevantes. Como muestra de lo contrario, es interesante la investigación previa (Whitehill y Ciocca, 2000a) que el equipo de Tara Whitehill realiza antes de construir su test de inteligibilidad para el chino cantonés (Whitehill y Ciocca, 2000b) y el descubrimiento del importante papel que los contrastes prosódicos juegan en la inteligibilidad de las disartrias al tratarse de una lengua tonal.

Desdichadamente, no contamos en castellano con una tradición de estudio que hoy pudiera proporcionarnos datos de partida acerca de los contrastes fonético-articulatorios más vulnerados en las disartrias. En este contexto de sequía hay que mencionar la valiosa aportación de Brancal y Ferrer (1998), si bien se basa sólo sobre un grupo diagnóstico específico (ataxias hereditarias) y sin el nivel de exhaustividad que requeriría la construcción de un test de inteligibilidad para las disartrias en castellano. En este sentido, la Universidad Jaume I de Castellón ha iniciado un proyecto destinado a conocer los errores más frecuentes en disártricos de habla castellana como paso preliminar para la construcción de un test de inteligibilidad.

\section{Caracterización acústica de la inteligibilidad}

Cada vez hay mayor empeño por caracterizar objetivamente y de forma precisa aquellos rasgos acústicos que son los principales responsables del deterioro de la inteligibilidad del 
lenguaje. Esta tarea se ha visto facilitada por el creciente desarrollo y asequibilidad de los equipos de análisis acústicos del habla.

La investigación sobre la señal de habla disártrica y su comparación con el habla normal ha puesto de manifiesto algunos correlatos acústicos de naturaleza patológica que podrían ayudar a explicar el deterioro de la inteligibilidad. Los esfuerzos se encaminan, además, a poner en relación estos correlatos acústicos, que pueden ser medidos cuantitativamente con precisión, con la información aportada por los juicios perceptivos y las tareas de identificación. Se trata, por tanto, de un enfoque complementario entre el plano acústico y el perceptivo.

El análisis acústico del habla disártrica se dirige a los dos subsistemas básicos de la producción de lenguaje (Kent, Weismer, Kent, Vorperian, y Duffy, 1999): fuente sonora o voz y función del tracto vocal. En el primero cobra importancia el examen de la prosodia a través de los valores que adopta la Frecuencia fundamental de la voz (Fo). Por otra parte, se está comenzando a aplicar las herramientas de análisis de la voz patológica al campo concreto de las disartrias. Los primeros estudios (Kent, Vorperian, y Duffy, 1999; Kent, Vorperian, Kent, y Duffy, 2003) ofrecen perspectivas muy prometedoras, particularmente las que se basan en parámetros de voz de naturaleza multidimensional. Entre los instrumentos de análisis destacaríamos el MDVP Multi-Dimensional Voice Program de la casa Kay Elemetrics que ofrece un repertorio de treinta parámetros clasificados en siete dimensiones, de probada utilidad y fiabilidad en el análisis de la voz. Una parte importante de la investigación sobre la voz hecha en las universidades de Castellón y Valencia se ha basado precisamente en dicho instrumento (González y Cervera, 2001; González, Cervera y Miralles, 2002; González, Cervera y Llau, 2003).

El estudio de la función del tracto vocal tiene una incidencia más directa en la inteligibilidad al guardar relación con los movimientos articulatorios del lenguaje. Las 
herramientas de análisis básicas son la representación de la propia onda sonora y, sobre todo, los espectrogramas, que hoy pueden obtenerse no sólo mediante costosos equipos de análisis (como por ejemplo, el CSL o Computerized Speech Laboratory de Kay Elemetrics), sino a través de excelentes programas disponibles en internet de forma libre para fines no comerciales; por ejemplo, el PRAAT de la Universidad de Amsterdam (Boersma \& Weenink, 1996), el TF32 de la Universidad Wisconsin-Madison, que es la versión actualizada del conocido y muy usado CSpeech (Milenkovic, 1989), o el SFS de la Universidad de Londres (Huckvale et al., 1987).

El análisis conjunto de la onda y el espectrograma se realiza en las dos dimensiones básicas de todo análisis de sonido: temporal y frecuencial. En la dimensión temporal se estudia la velocidad o tasa de habla y las duraciones relativas entre sílabas y fonemas. Por ejemplo, en muchas formas de ataxias aparece una isocronía patológica que no diferencia entre la duración de sílabas acentuadas y no acentuadas y que contribuye a la impresión de habla escandida típica de las disartrias cerebelosas. De forma particular, el análisis se centra también en parámetros temporales que son críticos en la identificación de ciertos fonemas, como es el caso del archiestudiado -en inglés fundamentalmente- VOT (Voice Onset Time), o tiempo entre la liberación de aire de las consonantes oclusivas y el comienzo de la vocal siguiente, que es clave en la distinción sonora-sorda.

En la dimensión frecuencial son primordiales las resonancias del conducto vocal que resultan de las diversas configuraciones que éste va adoptando en tiempo real, producto de los movimientos articulatorios. El análisis de estas resonancias o formantes tiene una gran importancia por su directa implicación en la inteligibilidad del habla. De hecho, el equipo de Wisconsin-Madison encuentra una relación muy directa entre inteligibilidad y la trayectoria frecuencial de los dos primeros formantes (F1 y F2), particularmente de F2 (Weismer y Martin, 1992). En muchas disartrias la inteligibilidad se deteriora porque el paciente no llega 
a alcanzar las posiciones articulatorias propias de cada fonema y esto se traduce en valores alterados de los formantes vocálicos y trayectorias formánticas planas y uniformes en las transiciones entre consonantes y vocales (Weismer, Martin, Kent, y Kent, 1992). Otros rasgos acústicos relevantes para la inteligibilidad son las propiedades del ruido fricativo (distribución frecuencial, centro de gravedad, etc.) de muchas consonantes y los rasgos de nasalidad (ancho de banda y energía de los formantes, presencia de murmuro nasal, etc.), que reflejan la función del velo del paladar.

A efectos ilustrativos presentamos (Figura 1) dos espectrogramas de banda ancha correspondientes a un mismo fragmento de habla ("la isla", como parte de la frase "la isla está en medio del lago"), pronunciado por dos mujeres de la misma edad (44 años). Una de ellas (A) tiene el habla normal, mientras que la otra (B) sufre disartria cerebelosa causada por una ataxia de Friedreich de veinte años de evolución. Comparando ambos espectrogramas se observa, en primer lugar, la tasa más lenta del habla patológica, al ocupar 910 milisegundos el mismo fragmento que dura 435 en el habla normal. Se observa, además, que la trayectoria de los dos primeros formantes ( $\mathrm{F} 1$ y F2) es más plana y uniforme en la mujer con disartria, como resultado de la hipoarticulación causada por el daño neurológico. Obsérvese la práctica ausencia del ruido fricativo de la /s/, al no alcanzar la lengua la posición requerida para su producción. En líneas generales, el segundo espectrograma tiene un nivel superior de ruido con una estructura periódica (estrías verticales que indican los movimientos de abertura y cierre de las cuerdas vocales) menos clara. Los fonemas aparecen menos diferenciados: por ejemplo, no es visible la clara y brusca distinción, propia del habla normal, entre el fonema lateral /1/ y la vocal /a/, resultado de la rápida retirada de la lengua de su posición alveolar. Finalmente, son visibles abundantes crepitaciones de inicio, o movimientos de contacto, generalmente velar, que suelen acompañar a muchos tipos de disartrias. 


\section{Conclusión}

Entre los múltiples efectos que puede causar el daño neurológico en el lenguaje, destaca, por su centralidad en el proceso comunicativo, la pérdida de inteligibilidad que con frecuencia acompaña en mayor o menor grado al habla disártrica. Una medida válida y fiable de la misma no es fácil, al tratarse de una dimensión compleja y al mismo tiempo de naturaleza relativa, sometida al influjo de múltiples variables procedentes tanto del propio hablante, como del oyente y el canal (ambiente físico). Sin embargo, la necesidad de instrumentos de medida adecuados se hace sentir tanto desde el punto de vista diagnóstico, como en la evaluación del progreso de los tratamientos (o enfermedad).

La investigación en otros ámbitos lingüísticos -sobre todo el anglosajón- revela la utilidad de un enfoque analítico-explicativo que, al estilo del test de Kent (Kent et al., 1989), vaya más allá de la obtención de un único índice global de severidad e intente explicar las causas del deterioro de la inteligibilidad. Hoy falta para el castellano un test normativizado, con un conjunto de estímulos cuidadosamente seleccionados para medir y analizar en detalle la inteligibilidad del habla disártrica. Incluso falta investigación previa que descubra cuáles son los contrastes fonético-articulatorios de nuestra lengua más vulnerables al daño neurológico. Se trataría de un paso previo indispensable para establecer el repertorio de contrastes que, entre otros aspectos del lenguaje, deben ser incluidos en un test de esta índole. En este sentido, la Universidad Jaume I de Castellón ha iniciado un proyecto de investigación (I+D, 2003) para cubrir esta laguna, hallándose ahora en la fase inicial de creación de un corpus de habla patológica correspondiente a los distintos tipos de disartria. 


\section{REFERENCIAS}

Auzou, P., Özsancak, C., Jan, M., Léonardon, S., Ménard, J.F., Gaillard, M.J., Eustache, F., y Hannequin, D. (1998). Evaluation clinique de la Dysarthrie: présentation et validation d'une méthode. Rev Neurol (Paris), 154, 523-530.

Beukelman, D.R. y Yorkston, K.M. (1979). The relationship between information transfer and speech intelligibility of dysarthric speakers. Journal of Communication Disorders, 12, 189196.

Boersma, P. y Weenink, D. (1996). Praat: A system for doing phonetics by computer. Technical Report 132, Institute of Phonetic Science of the University of Amsterdam. Disponible en http://www.fon.hum.uva.nl/praat/.

Brancal, M y Ferrer, A. (1998). Análisis perceptual de las características del habla en personas afectas de ataxias hereditarias. Revista de Logopedia, Foniatría y Audiología, 18, 213-224.

Cárdenas, R. y Marrero, V. (1994). Cuaderno de logoaudiometría. Cuadernos de la UNED. Madrid: UNED.

Connolly, J.H. (1986). Intelligibility: a linguistic view. British Journal of Disorders of Communication, 21, 371-376.

Darley, F., Aronson, A., y Brown, J. (1975). Motor Speech Disorders. Philadelphia: Saunders. (trad. Cast. 1978).

Dowden, P.A., Yorkston, K.M., y Stoel-Gammon, C. (1987). Speech intelligibility of augmented system users: Effects of context. American Speech and Hearing Association, 29, 10.

Duffy, J.R. (1995). Motor Speech Disorders. Substrates, Differential Diagnosis and Management. St Louis, MO: Mosby

Enderby, P. (1983). Frenchay Dysarthria Assessment. Austin, TX: Pro-Ed. 
Flanagan, J.L. (1972). Speech analysis, syntheis, and perception. New York: Springer-Verlag.

Fletcher, H. y Allen, J. (1995). ASA Edition of Speech and Hearing in Comunication. Sewickley, PA: Acoustical Society of America. (orig. 1953)

Gentil, M. (1992). Phonetic intelligibility testing in dysarthria. Clinical Linguistics and Phonetics, 6, 179-189.

González, J. y Cervera, T. (2001). The effect of MPEG audio compression on a set of multidimesional voice parameters. Logopedics Phoniatrics \& Vocology, 26, 124-138.

González, J., Cervera, T. y Miralles, J.L. (2002). Análisis acústico de la voz: Fiabilidad de un conjunto de Parámetros Multidimensionales. Acta Otorrinolaringológica Española, 53, 256-268.

González, J., Cervera, T., y Llau, M.J. (2003). Acoustic analysis of patological voices compressed with MPEG system. Journal of voice, 17, 1-14.

Guildford, J.P. (1954). Psychometric Methods. New York: McGraw-Hill.

Huckvale, M.A., Brookes, D.M., Dworkin, L.T., Johnson, M.E., Pearce, D.J., y Whitaker, L. The SPAR Speech Filing System. European Conference on Speech Technology, Edinburgh, 1987. Disponible en http://www.phon.ucl.ac.uk/resource/sfs/.

Kent, R.D. (1992). Intelligibility in speech disorders: Theory, measurement and management. Amsterdam: Benjamins Pub.

Kent, R.D, Vorperian, H.K., y Duffy, J.R. (1999). Reliability of the Multi-Dimensional Voice Program for the analysis of voice samples of subjects with dysarthria. American Journal of Speech-Language Pathology, 8,129-136.

Kent, R.D., Vorperian, H.K., Kent, J.F., y Duffy, J.R. (2003). Voice dysfunction in dysarthria: application of the Multi-Dimensional Voice Program. Journal of Communication Disorders, 36,281-306. 
Kent, R., Weismer, G., Kent, J., y Rosenbeck, J. (1989). Toward explanatory intelligibility testing in dysarthria. Journal of Speech and Hearing Disorders, 54, 482-499.

Kent, R.D., Weismer, G., Kent, J.F., Vorperian, H.K., y Duffy, J.R. (1999). Acoustic studies of dysarthric speech: Methods, progress, and potential. Journal of Communication Disorders, 32,141-186.

Melle, N. (2003). Disartria en el daño cerebral adquirido: hacia un método global de evaluación. Revista de Logopedia, Foniatría y Audiología, 23, 20-29.

Milenkovic, P. (1989). Cspeech 3.0 (Speech Analysis Software Program, University of Wisconsin, 1415, Johnson Drive, Madison, WI 53706). Disponible en http://userpages.chorus.net/cspeech/.

Miller, N. (2001). Disartrias. En: M. Puyuelo (ed.). Casos clínicos en logopedia, vol 3. Barcelona: Masson.

Monfort, M., Juárez, A. (2001). Test de inteligibilidad. Madrid: Entha.

Platt, L.J., Andrews, G., Young, M., y Quinn, P. (1980). Dysarthria of adult cerebral palsy: I. Intelligibility and articulatory impairment. Journal of Speech and Hearing Research, 23, $28-40$.

Robertson, S.J. (1982). Robertson Dysarthria Profile. Bicester: Winslow Press.

Samar, V.J., Metz, D.E. (1988) Criterion validity of speech intelligibility rating-scale procedures for the hearing-impaired population. Journal of Speech and Hearing Research, 31, 307-316.

Schiavetti, N., Metz, D.E., Sitler, R.W. (1981). Construct validity of direct magnitude estimation and interval scaling of speech intelligibility: Evidence from a study of the hearing impaired. Journal of speech and Hearing Research, 24, 441-445. 
Schiavetti, N. (1992). Scaling procedures for the measurement of speech intelligibility. En R.D. Kent (ed.) Intelligibility in Speech Disorders (pp. 11-34). Amsterdam: Benjamins Pub.

Snodgrass, J.G. (1975). Psychophysics. En B. Scharf (ed.) Experimental Sensory Psychology, (pp 17-67). Glenview, IL: Scott Foresman.

Stevens, S.S (1975). Psychophysics. New York: Wiley.

Tato, J.M. (1949). Características acústicas de nuestro idioma. Revista de Otolaringología, 1, 17-34.

Tikofsky, R.S. (1970). A revised list for the estimation of dysarthric single word intelligibility. Journal of Speech and Hearing Research, 7, 59-64.

Tikofsky, R.S. y Tikofsky, R.P. (1964). Intelligibility measures of dysarthric speech. Journal of Speech and Hearing Research, 7, 325-333.

Villalba, A., Ferrández, J.A., Ros, V. (1999). Listas de palabras para evaluar la percepción del lenguaje oral en niños sordos e hipoacúsicos. Acta Otorrinolaringológica Española, 50, 151-157.

Weismer, G., Martin, R. (1992). Acoustic and perceptual approaches to the study of intelligibility. En R.D. Kent (ed.) Intelligibility in Speech Disorders (pp. 67-118). Amsterdam: Benjamins Pub.

Weismer, G., Martin, R., Kent, R.D., Kent, J.F. (1992). Formant trajectory characteristics of males with amyotrophic lateral sclerosis. Journal of Acoustic Society of America, 91,108598.

Whitehill, T.L. y Ciocca, V. (2000a). Speech errors in Cantonese speaking adults with cerebral palsy. Clinical Linguistics and Phonetics, 14, 111-130. 
Whitehill, T.L. y Ciocca, V. (2000b). Perceptual-phonetics predictors of single-word intelligibility: A study of Cantonese Dysarthria. Journal of Speech, Language, and Hearing Research, 43, 1451-1465.

Yorkston, K.M., Beukelman, D.R. (1978). A comparison of techniques for measuring intelligibility of dysarthirc speech. Journal of Communication Disorders, 11, 499-512..

Yorkston, K.M., Beukelman, D.R. (1981). Communication efficiency of dysarthrics speakers as measured by sentence intelligibility and speaking rate. Journal of Speech and hearing disorders, 46, 296-301.

Yorkston, K.M., Beukelman, D.R. (1981). Assessment of Intelligibility of Dysarthric Speech. Austin, TX: Pro-Ed.

Yorkston, K.M., Beukelman, D.R. y Traynor, C.D. (1984). Computerized Assessment of Intelligibility of Dysarthric Speech (CAID). Austin, TX: Pro-Ed.

Yorkston, K.M., Dowden, P.A., Beukelman D.R. (1992) Intelligibility measurement as a tool in the clinical management of dysarthric speakers. En R.D. Kent (ed.) Intelligibility in Speech Disorders (pp. 11-34). Amsterdam: Benjamins Pub

Ziegler, W., Hartmann, E. (1993). Das Muenchener Verstandlinchkeistprofil. Nevernartz, 64, 653-658.

Ziegler, W., Hartmann, E, von Cramon, D. (1988). Word identification testing in the diagnostic evaluation of dysarthric speech. Clinical Linguistics and Phonetics, 2, 291-308. 

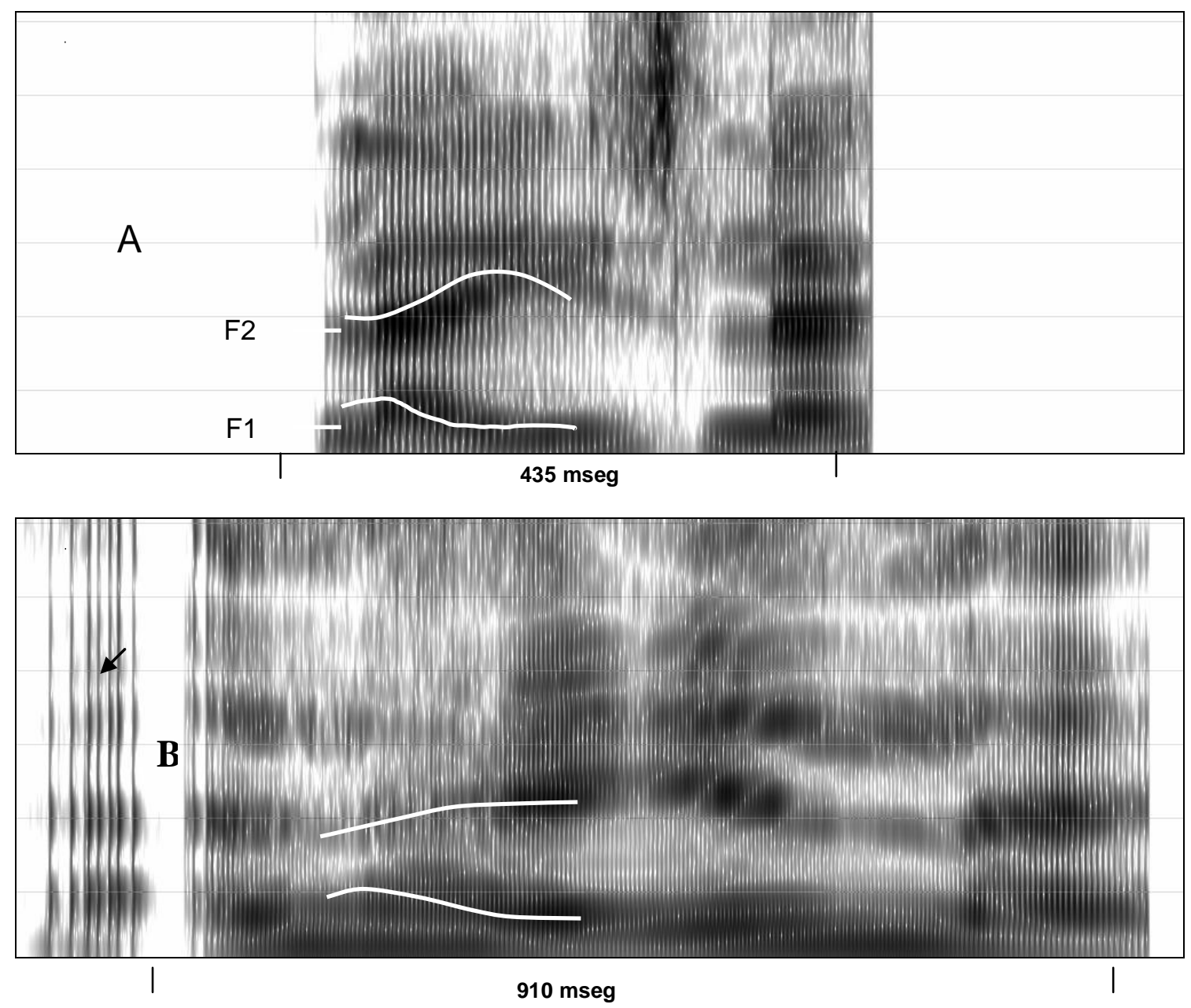

Figura 1: Espectrogramas de banda ancha (rango 0-6000 Hz.) del fragmento "la isla" extraído de una misma frase pronunciada por una mujer de habla normal (A) y una mujer con ataxia de Friedreich (B). Se han señalado con líneas blancas porciones del primer (F1) y segundo (F2) formantes. Algunos rasgos patológicos son: habla lenta (duración total de 910 milisegundos frente a 435); trayectorias de F1 y F2 más planas y uniformes, con menor diferenciación entre fonemas; voz con exceso de ruido; crepitación frecuente al iniciar la fonación (flecha). (Fuente: Base de voces de la Universidad Jaume I de Castellón). 
Tabla1. Instrumentos de medida de la inteligibilidad del habla disártrica basados en tareas de identificación.

\begin{tabular}{|c|c|c|c|c|c|c|}
\hline Instrumento & Autores & Fecha & Lengua & Estímulos & Tipo de medida o información & Muestra de estudio \\
\hline & $\begin{array}{l}\text { Tykofsky, R.S. y } \\
\text { Tykofsky, R.P. }\end{array}$ & 1964,1970 & Inglés & 50 palabras & Porcentaje de palabras correctas & 9 sujetos disártricos \\
\hline & Platt, L.J. et al. & $1978 / 1980$ & Inglés & $50 / 40$ palabras & Porcentaje de palabras correctas & 50 sujetos con parálisis cerebral \\
\hline $\begin{array}{l}\text { Assesment of } \\
\text { Intelligibility of } \\
\text { Dysarthric Speech }\end{array}$ & $\begin{array}{l}\text { Yorkston, K.M. } \\
\text { Beukelman, D.R. }\end{array}$ & $1981 / 1984$ & Inglés & $\begin{array}{l}50 \text { Palabras } \\
100 \text { Frases }\end{array}$ & $\begin{array}{l}\text { Índice cuantitativo de severidad } \\
\text { Palabras inteligibles por minuto } \\
\text { Ratio de comunicación efectiva } \\
\text { Tasa de habla }\end{array}$ & 13 sujetos disártricos \\
\hline $\begin{array}{l}\text { Robertson } \\
\text { Dysarthria Profile }\end{array}$ & Robertson, S.J. & 1982 & Inglés & $\begin{array}{l}\text { Palabras } \\
\text { Frases } \\
\text { Párrafo } \\
\text { Conversación } \\
\end{array}$ & $\begin{array}{l}\text { Porcentaje de palabras y frases correctas } \\
\text { Escalas para conversación }\end{array}$ & \\
\hline $\begin{array}{l}\text { Frenchay } \\
\text { Dysarthria } \\
\text { Assesment }\end{array}$ & Enderby, $\mathrm{P}$. & 1983 & Inglés & $\begin{array}{l}50 \text { Palabras } \\
25 \text { pares de } \\
\text { Frases } \\
\text { Conversación }\end{array}$ & $\begin{array}{l}\text { Porcentajes de palabras y frases correctas } \\
\text { en cinco intervalos } \\
\text { Escalas para conversación }\end{array}$ & 85 sujetos disártricos \\
\hline \multirow[t]{2}{*}{$\begin{array}{l}\text { Word intelligibility } \\
\text { Test }\end{array}$} & Kent,. D. & 1989 & Inglés & 70 palabras & $\begin{array}{l}\text { Índice de severidad. } \\
\text { Porcentaje de palabras correctas. } \\
\text { Análisis fonéticos de los errores. } \\
\text { (19 contrastes fonéticos) }\end{array}$ & $\begin{array}{l}13 \text { y } 25 \text { sujetos con Esclerosis } \\
\text { Lateral Amiotrófica }\end{array}$ \\
\hline & $\begin{array}{l}\text { Ziegler, W. } \\
\text { Hartman, E. } \\
\text { Von Cramon, D. }\end{array}$ & 1988 & Alemán & $\begin{array}{l}100 \text { conjuntos } \\
\text { de } 6 \text { palabras }\end{array}$ & Porcentaje de palabras correctas & 32 sujetos disártricos \\
\hline \multirow[t]{2}{*}{$\begin{array}{l}\text { Münchner } \\
\text { Verständlichkeits } \\
\text { Profil (MVP) }\end{array}$} & $\begin{array}{l}\text { Ziegler, W. } \\
\text { Hartman, E. }\end{array}$ & 1993 & Alemán & $\begin{array}{l}4 \text { listas de más } \\
\text { de } 100 \\
\text { palabras }\end{array}$ & Porcentaje de palabras correctas & 101 sujetos disártricos \\
\hline & Gentil, M. & 1992 & Francés & 72 palabras & $\begin{array}{l}\text { Índice severidad } \\
\text { Análisis fonéticos de los errores } \\
\text { (17 contrastes fonéticos) }\end{array}$ & $\begin{array}{l}9 \text { sujetos con Ataxia de } \\
\text { Friedreich. }\end{array}$ \\
\hline $\begin{array}{l}\text { Evaluation Clinique } \\
\text { de la Dysarthrie } \\
(E C D)\end{array}$ & Auzou, P. et al. & 1998 & Francés & $\begin{array}{l}\text { Palabras } \\
\text { Frases } \\
\text { Conversación }\end{array}$ & $\begin{array}{l}\text { Porcentaje para frases y palabras } \\
\text { Medida global para conversación }\end{array}$ & 15,18, y 100 sujetos disártricos \\
\hline $\begin{array}{l}\text { Cantonese Single- } \\
\text { Word Inteligibility } \\
\text { (CSIT) }\end{array}$ & $\begin{array}{l}\text { Whitehill, T y } \\
\text { Ciocca, V. }\end{array}$ & 2000 & $\begin{array}{l}\text { Chino } \\
\text { cantonés }\end{array}$ & 75 palabras & $\begin{array}{l}\text { Porcentaje de palabras correctas } \\
\text { (17 contrastes fonéticos) }\end{array}$ & 20 sujetos con parálisis cerebral \\
\hline
\end{tabular}

